\title{
ASSESSMENT AND COMPARISON OF MULTIVARIATE PROCESS CAPABILITY INDICES IN CERAMIC INDUSTRY
}

\author{
Md. Abu Zahid and Arifa Sultana \\ Department of Industrial and Production Engineering \\ Bangladesh University of Engineering and Technology, Dhaka-1000.
}

\begin{abstract}
Process capability indices are intended to provide a single-number assessment of the consistency of a manufacturing process relative to the engineering specification limits on quality characteristics. In many industrial instances product quality depends on a multitude of dependent characteristics and as a consequence, attention on capability indices shifts from univariate domain to multivariate domain. In this paper five different multivariate methodologies are used for measuring and comparing capability of a ceramic table-ware manufacturing process. Based on their multivariate process capability index values and expected rejection rate, the result shows that Castagliola's index is the best and followed by Chen, Taam, Shahriari and Braun respectively in this case.
\end{abstract}

Keywords: Process capability indices, Multivariate process capability indices, Multivariate Capability Plot, Multivariate control chart

\section{INTRODUCTION}

Process capability refers to the inherent ability of a process to produce similar parts for a sustained period of time under a given set of conditions when operating in a state of statistical control. Process capability indices (PCIs), are used to provide a numerical measure of whether a production process is capable of producing items satisfying the quality requirement present in the factory.

When quality of a product depends upon single quality characteristic, the simplest form of the process capability index (PCI) is the ratio of the specification width to the process spread. However, it can be often observed that quality of a product does not depend only on a single quality characteristic; rather it depends on more than one quality characteristic. Thus multivariate process capability indices (MPCIs) arise to produce a single number representing capability for two or more quality variables.

Multivariate capability indices usually produce one number jointly representing capability for two or more quality variables. Generally multivariate process capability indices can be obtained from a number of different methods. Among them five methods: (a) the ratio of a specification limit to process variation or modified process variation, (b) the probability of nonconforming products over rectangular tolerance zone, (c) implementing loss functions and vector representation, (d) theoretical proportion of non-conforming products over convex polygons and (e) global approach viewing multivariate quality control. Taam, Subbaiah, and Liddy ${ }^{1}$ generated the first multivariate capability index. $\mathrm{Chen}^{2}$ also proposes a method in order to estimate the multivariate $\mathrm{C}_{\mathrm{p}}$ using a non-conforming proportion approach. Shahriari et al. ${ }^{3}$ and Wang et al. proposed a process capability multivariate vector in order to evaluate the process performance. Lorenz Braun ${ }^{4}$ defined $C_{p}$ and $C_{p k}$ as $E C_{p}$ and $E C_{p k}$, where the both multivariate process region and the multivariate tolerance region are of elliptical shape. Philippe Castagliola and Jose-Victor Castellanos ${ }^{5}$ defined two new capability indices $B C_{p}$ and $B C_{p k}$ dedicated to two quality characteristics, based on the computation of the theoretical

\begin{tabular}{|c|c|c|c|}
\hline \multicolumn{4}{|c|}{ Nomenclature: } \\
\hline $\operatorname{det}()$. & determinant & $\alpha$ & percentage of allowable nonconformance \\
\hline $\mathrm{F}_{(\mathrm{v}, \mathrm{n}-\mathrm{v})}$ & Snedecor's F distribution with $\mathrm{v}$ and $\mathrm{n}-\mathrm{v}$ degrees & $\beta$ & beta function \\
\hline $\mathrm{LPL}_{\mathrm{i}}$ & $\begin{array}{l}\text { freedom } \\
\text { lower process limit (modified) for variable } i, i= \\
1,2\end{array}$ & $\begin{array}{l}\chi_{(v, \alpha)}^{2} \\
\phi(x)\end{array}$ & $\begin{array}{l}\text { upper } 100(\alpha) \% \text { of a } \chi^{2} \text { distribution } \\
\text { probability density function for normal }\end{array}$ \\
\hline $\begin{array}{l}\text { LSL }_{\mathrm{i}} \\
\text { MPCIs }\end{array}$ & $\begin{array}{l}\text { lower specification limit for variable } \mathrm{i}, \mathrm{i}=1,2 \\
\text { Multivariate process capability indices }\end{array}$ & $\Phi(\mathrm{x})$ & $\begin{array}{l}\text { distribution } \\
\text { cumulative distribution function for normal }\end{array}$ \\
\hline $\begin{array}{l}\text { PCIs } \\
\mathbf{R}\end{array}$ & $\begin{array}{l}\text { Process capability indices } \\
\text { rotation matrix }\end{array}$ & $\Gamma$ & $\begin{array}{l}\text { distribution } \\
\text { gamma function }\end{array}$ \\
\hline $\begin{array}{l}\mathbf{K} \\
\mathrm{S}\end{array}$ & $\begin{array}{l}\text { rotation matrix } \\
\text { sample covariance matrix }\end{array}$ & $\vec{\mu}_{0}$ & vector of target \\
\hline $\mathrm{S}^{-1}$ & inverse of sample covariance matrix & $\rho$ & correlation coefficient \\
\hline sgn & signum function & $\sigma_{1}$ & standard deviation for variable $\mathrm{x}_{1}$ \\
\hline $\mathrm{T}^{2}$ & Hotelling's T-square statistic & $\sigma_{2}$ & standard deviation for variable $\mathrm{x}_{2}$ \\
\hline $\mathrm{UPL}_{\mathrm{i}}$ & $\begin{array}{l}\text { upper process limit (modified) for variable } \mathrm{i}, \mathrm{i} \\
=1,2\end{array}$ & $\sigma_{12}$ & covariance between variable $x_{1}$ and $x_{2}$ \\
\hline $\mathrm{USL}_{\mathrm{i}}$ & upper specification limit for variable $\mathrm{i}, \mathrm{i}=1,2$ & $\begin{array}{l}\Sigma \\
\Sigma^{-1}\end{array}$ & $\begin{array}{l}\text { covariance matrix } \\
\text { inverse of covariance matrix }\end{array}$ \\
\hline $\mathrm{v}$ & $\begin{array}{l}\text { number of quality characteristics considered, } \\
\text { here } v=2\end{array}$ & $\Sigma_{\mathrm{i}}^{-1}$ & a matrix obtained from $\Sigma^{-1}$ by deleting the $\mathrm{i}^{\text {th }}$ row \\
\hline $\mathbf{V}$ & vector of eigenvalues & $\Sigma^{t h}$ & $\begin{array}{l}\text { and column } \\
\text { hypothetical variance matrix transpose of a }\end{array}$ \\
\hline$\overline{\mathbf{X}}$ & vector of mean & $2_{y y}$ & $\begin{array}{l}\text { nypotnetical variance matrix transpose or a } \\
\text { matrix }\end{array}$ \\
\hline
\end{tabular}


proportion of non-conforming products over convex polygons. For the bivariate case $\mathrm{Pal}^{6}$ proposed an index. Bothe ${ }^{7}$ proposed a method in order to compute the multivariate $\mathrm{C}_{\mathrm{pk}}$ index. Wang and $\mathrm{Chen}^{8}$ proposed multivariate equivalents for $C_{p}, C_{p k}, C_{p m}$ and $C_{p m k}$ based on the PCA (Principal Component Analysis) decomposition. Wang and $\mathrm{Du}^{9}$ proposed the same indices and one extension to the non-normal multivariate case.

Process capability index is not commonly used as Statistical Process Control (SPC) tool to assess capability of the process and to produce quality of the products in manufacturing arena of Bangladesh However, this single number assessment can be a popular SPC tool for assessing ability of the process to meet specification limit.

Ceramic industry is one of the exporting industrial sectors in Bangladesh. The management of some of the ceramic industries claimed that, they had $30-40 \%$ rejection while producing the product. The rejection can be occurred mainly due to the manufacturing process they are using and/or material they are using for the product and/or by the man involved in the process. Among these industries, Shinepukur Ceramic Industry has been chosen for measuring process capability index. The focus of this paper is to check the manufacturing process to meet the management requirement by using process capability indices.

The objective of the present work is to compute and compare multivariate process capability indices for ceramic cup (can cup S-9) manufacturing process in Shinepukur ceramic industry (porcelain section) using five different methods
i. Taam's method (1993)
ii. Chen's method (1994)
iii. Shahriari's method (1995)
iv. Lorenz Braun's Method (2001)
v. Castagliola's Method (2005)

Final objective is to decide which one among these multivariate methods is the best suited for ceramic manufacturing.

All the information were gathered together and analyzed to predict the extent to which the process was able to hold tolerance or customer requirements and which one of the multivariate methods was the best suited for the selected ceramic cup manufacturing process.

For the test of multivariate normality and multivariate statistical in-control situation the software: STAT GRAPHICS CENTURION XV is used. For mathematical computation and multivariate plotting the mathematical algorithms: MATLAB 7.1 and MATHEMATICA 5 are used.

\section{OVERVIEW OF SELECTED MULTIVARIATE PROCESS CAPABILITY INDICES}

\subsection{Taam's Method}

Taam et al. defined a multivariate capability index as a ratio of the volume of the modified tolerance region $\left(\mathrm{R}_{1}\right)$ to the volume of $99.73 \%$ process region $\left(\mathrm{R}_{2}\right)$.

$$
\mathrm{MC}_{\mathrm{pm}}=\frac{\text { Volume of } \mathrm{R}_{1}}{\text { Volume of } \mathrm{R}_{2}}
$$

If the process data are multivariate normal, then $R_{2}$ is an elliptical region. The modified tolerance region is the largest ellipsoid completely within the engineering tolerance region and centered at the target. The estimate of $\mathrm{MC}_{\mathrm{pm}}$ can be calculated as, where $\quad \mathrm{MC}_{\mathrm{pm}}=\frac{\hat{\mathrm{C}}_{\mathrm{p}}}{\hat{\mathrm{D}}}$

$$
\begin{aligned}
\hat{\mathrm{C}}_{\mathrm{p}} & =\frac{\text { vol.(tolerance region) }}{\text { vol.(estimated } 99.73 \% \text { process region })} \\
& =\frac{\text { vol.(tolerance region) }}{|\mathrm{S}|^{1 / 2}(\pi \mathrm{K})^{\mathrm{v} / 2}[\Gamma(\mathrm{v} / 2+1)]^{-1}} \\
\hat{D} & =\left[1+\frac{n}{n-1}\left(\overline{\mathbf{X}}-\mu_{0}\right)^{\prime} \mathbf{S}^{-1}\left(\overline{\mathbf{X}}-\mu_{0}\right)\right]
\end{aligned}
$$

Here, $\mathrm{K}$ is the $99.73 \%$ quantile of a $\chi^{2}$ distribution. When the process mean vector equals the target vector, and the index has the value 1 , then $99.73 \%$ of the process values lie within the modified tolerance region.

\subsection{Chen's Method}

Chen proposed this method, which defines multivariate capability index for rectangular tolerance zone. Firstly a general tolerance zone is defined as

$$
V=\left\{x \in R^{v}: h(x-T) \leq r_{o}\right\},
$$

where $\mathrm{h}(\mathrm{x})$ is specific positive function with the same scale as $\mathrm{x}, T \in R^{v}$ is a constant vector and $\mathrm{r}_{0}$ a positive number. Then a rectangular solid tolerance zone is defined by

$$
V=\left\{x \in R^{v}:\left|x_{i}-T_{i}\right| \leq r_{i}, i=1, \ldots v\right\},
$$

Where, $T_{i}$ and $r_{i}$ are specific constants. Another expression for $\mathrm{V}$ is

$$
V=\left\{x \in R^{v}: \max \left\{\left|x_{i}-T_{i}\right| / r_{i}, i=1, \ldots, v\right\} \leq 1\right\}
$$

Thus, $\mathrm{V}$ has the structure,

$$
h(x)=\max \left\{\left|x_{i}\right| / r_{i}, i=1, \ldots, v\right\} .
$$

Consequently, the multivariate $\mathrm{PCI}$

where $\mathrm{r}$ is such that

$$
\mathrm{MC}_{\mathrm{P}}=1 / \mathrm{r}
$$

$P\left(\max \left\{\left|X_{i}-T_{i}\right| / r_{i}, i=1, \ldots, v\right\} \leq r\right)=1-\alpha$

Let $\mathrm{F}$ be the cumulative distribution function of $\mathrm{h}(\mathrm{X}$ T). Then $r=F^{-1}(1-\alpha)$,i.e., the $100(1-\alpha)$-th percentile of F. It follows immediately that for any $\mathrm{y}>0$,

$$
F(y) \leq \min \left\{P\left(\left|X_{i}-T_{i}\right| \leq r_{i} y\right), i=1, \ldots, v\right\}
$$

So, a necessary condition for a process to be capable over rectangular solid zone is that each individual univariate process is capable with the corresponding specification limits.

As in this paper MPCIs should be computed for a bivariate process, Let $\mathrm{v}=2$ and $\Sigma=\left(\sigma_{\mathrm{ij}}\right)$, where $\sigma_{11}=\sigma_{1}^{2}, \sigma_{22}=\sigma_{2}^{2}$ and $\sigma_{12}=\rho \sigma_{1} \sigma_{2}$. Then

$$
F(y)=P\left(\left|\frac{X_{1}-T_{1}}{r_{1}}\right| \leq y,\left|\frac{X_{2}-T_{2}}{r_{2}}\right| \leq y\right)=\int_{-\infty}^{\infty} g(u ; y) e^{-u^{2}} d u
$$

$$
\text { with } \begin{aligned}
g(u ; y) & =\pi^{-1 / 2} \prod_{i=1}^{2}\left\{\Phi\left(a_{i 1}\right)-\Phi\left(a_{i 2}\right)\right\}, \text { where } \\
a_{i 1}= & \frac{\operatorname{sgn}^{i-1}(\rho) \sqrt{2|\rho|} u+\left(r_{i} y-\delta_{i}\right) / \sigma_{i}}{\sqrt{1-|\rho|}}, \\
a_{i 2} & =\frac{\operatorname{sgn}^{i-1}(\rho) \sqrt{2|\rho|} u-\left(r_{i} y+\delta_{i}\right) / \sigma_{i}}{\sqrt{1-|\rho|}},
\end{aligned}
$$


and $\delta_{i}=\mu_{i}-T_{i}, i=1,2$. The function $\mathrm{F}(\mathrm{y})$ can be numerically realized by utilizing computer algorithms.

Then $r=F^{-1}(1-\alpha)$, hence $M C_{p}=\frac{1}{r}$.

\subsection{Shahriari's Method}

This method was proposed by Shahriari et al. It consists of a vector with three components.

The first component is the measurement of capability. For bivariate normal process the process region is an elliptical contour. The modified tolerance region is the smallest rectangle around this ellipse. The arms of the rectangle are the lower and upper process limits $\left(\mathrm{LPL}_{\mathrm{i}}\right.$ and $\mathrm{UPL}_{\mathrm{i}}$ respectively, $\left.\mathrm{i}=1,2, \ldots, \mathrm{v}\right)$. It can be determined by solving the equations of first derivatives, with respect to each $\mathrm{x}_{\mathrm{i}}$, of the quadratic form,

$$
\left(\boldsymbol{X}-\mu_{0}\right)^{\prime} \Sigma\left(\boldsymbol{X}-\mu_{0}\right)=\chi_{(v, \alpha)}^{2}
$$

In this equation when the process data is multivariate normal, the distribution of the statistic will follow $\chi^{2}$ distribution. Solving the equation the upper and lower limits become,

$$
\begin{aligned}
& U P L_{i}=\mu_{i}+\sqrt{\frac{\chi_{(v, \alpha)}^{2} \operatorname{det}\left(\Sigma_{i}^{-1}\right)}{\operatorname{det}\left(\Sigma^{-1}\right.}}, \\
& L P L_{i}=\mu_{i}-\sqrt{\frac{\chi_{(v, \alpha)}^{2} \operatorname{det}\left(\Sigma_{i}^{-1}\right)}{\operatorname{det}\left(\Sigma^{-1}\right.}}, \\
& i=1,2, \ldots, v,
\end{aligned}
$$

where $\chi^{2}(v, \alpha)$ is the upper $100(\alpha) \%$ of a $\chi^{2}$ distribution with $v$ degrees of freedom associated with the probability contour and $\operatorname{det}\left(\Sigma_{i}^{-1}\right)$ is the determinant of $\Sigma_{i}^{-1}$, a matrix obtained from $\Sigma^{-1}$ by deleting the $i$ th row and column. The concept is to construct a modified process region with the same general geometric shape as the engineering tolerance region. However, the size and relative dimension of its sides are determined by the probability contour. That is, the modified process region is not a proportional change in the engineering tolerance region. For this illustration,

$$
C_{p M}=\left[\frac{\prod_{i=1}^{v}\left(U S L_{i}-L S L_{i}\right)}{\prod_{i=1}^{v}\left(U P L_{i}-L P L_{i}\right)}\right]^{\frac{1}{v}} .
$$

The second component is the locations of centers (target and observation mean). A Hotelling $\mathrm{T}^{2}$ statistic is computed and the second component is defined as the significance level of the observed value. That is,

$$
T^{2}=n\left(\overline{\mathbf{X}}-\mu_{0}\right)^{\prime} \mathbf{S}^{-1}\left(\overline{\mathbf{X}}-\mu_{0}\right)
$$

With the second component defined as,

$$
\left.P V=P\left(T^{2}\right\rangle \frac{v(n-1)}{n-v} F_{(v, n-v)}\right)
$$

Where, $\mathrm{F}_{\mathrm{v}, \mathrm{n}-\mathrm{v}}$ is the Snedecor's $\mathrm{F}$ distribution with $\mathrm{v}$ and $\mathrm{n}$ $\mathrm{v}$ degrees of freedom. As PV is a probability value, it will never exceed 1; values close to zero indicate that the center of the process is far from the engineering target value.

The third component is summarizes a comparison of the locations of the regions (process region and modified tolerance region).

$$
L I= \begin{cases}1 & \begin{array}{l}
\text { if modified process region is } \\
\text { contained within the tolerance } \\
\text { regions } \\
\text { other wise }
\end{array} \\
0 & \text { whe }\end{cases}
$$

It indicates whether any part of the modified process region falls outside the engineering specifications. In summary this method has three components,

$$
\left[\mathrm{C}_{\mathrm{pM}}, \mathrm{PV}, \mathrm{LI}\right]
$$

representing a comparison of volumes of regions, locations of centers, and locations of regions.

\subsection{Braun's Method}

Lorenz Braun proposed this method which is an integral part of a global approach viewing multivariate quality control. In this method the tolerance rectangle is replaced by a tolerance ellipse, considering the dependency of the two quality characteristics. The surface of the tolerance ellipse or the volume of a tolerance (hyper-) ellipsoid is

$$
\text { Vol }_{T o l}=\left|\Sigma_{y y}^{t h}\right|^{1 / 2} \cdot\left(\chi_{m, 0.9973}^{2} \cdot \pi\right)^{m / 2} \cdot\left[\Gamma(m / 2+1]^{-1}\right.
$$

where $\mathrm{m}$ is the number of quality characteristics, $\Gamma$ corresponds to the gamma function and $\chi_{m, 0.9973}^{2}$ is the 0.9973 percentile of the $\chi^{2}$ distribution with $\mathrm{m}$ degrees of freedom. $\quad \Sigma_{y y}^{t h}$ is the hypothetical variance matrix of the quality characteristics, which fits with a given probability of 99.73 percent into the tolerance rectangle or in the tolerance (hyper-cube). The hypothetical variance matrix is calculated using the correlation matrix of the quality characteristics $\underline{R}_{y y}$ by

$$
\Sigma_{y y}^{\text {th }}=\operatorname{diag}\left(\frac{\underline{T}_{u}-\underline{T}_{l}}{6}\right) \underline{R}_{y y} \operatorname{diag}\left(\frac{\underline{T}_{u}-\underline{T}_{l}}{6}\right) .
$$

$\operatorname{diag}(. .$.$) is a matrix, whose elements beside its main$ diagonal elements are equal to zero and whose main diagonal elements are, corresponding to the $3 \sigma$ rule, the sixth part of the tolerance intervals of the quality characteristics. A Comparison of this volume with the volume of the process region (VolProz) is the basic of the elliptical process capability.

The basic elliptical process capability $E C_{p}$ compares the 99.73 per cent concentration ellipsoid (elliptical process region) of the vector $\underline{Y}^{T}=\left(Y_{1} \ldots, Y_{m}\right)$ of the quality characteristics with the tolerance ellipsoid (elliptical tolerance region). That $E C_{p}$ is

$$
\begin{aligned}
E C_{p} & =\left(\frac{\operatorname{Vol}_{T o l}}{\operatorname{Vol}_{\text {Proz }}}\right)^{1 / m} \\
& =\prod_{j=1}^{m}\left(C_{p . j}\right)^{1 / j} .
\end{aligned}
$$

$C_{p . j}$ thereby corresponds to the univariate basic process capabilities of the $\mathrm{m}$ quality characteristics. The basic elliptical process capability is not influenced by the dependency structure of the quality characteristics. This is also meaningful, since otherwise $E C_{p}$ would depend on the degree of multicollinearity. On the other hand, the deviation between the vector of expected values $\underline{M}^{T}=\left(M_{1}, \ldots, M_{m}\right)$ and the vector of the expected values 
$\mu^{T}=\left(\mu_{1}, \ldots, \mu_{m}\right)$ must consider the dependency structure of the quality characteristics. The factor of correction of the elliptical process capability $K_{E}$ and the corrected elliptical process capability are

$$
\begin{gathered}
K_{E}=\sqrt{\frac{(\underline{\mu}-\underline{M})^{T}\left(\Sigma_{y y}^{t h}\right)^{-1}(\underline{\mu}-\underline{M})}{\chi_{m, 0.9973}^{2}}} \\
E C_{p k}=E C_{p} \cdot\left(1-K_{E}\right)
\end{gathered}
$$

The numerator in the equation of $\mathrm{K}_{\mathrm{E}}$ corresponds to an elliptical equation. The denominator is the 0.9973 percentile of the $\chi^{2}$ distribution with $\mathrm{m}$ degrees of freedom. The further the vector of expected values withdraws from the vector of target values, the larger the counter becomes in relation to the denominator. If a value $K_{E}$ of 1 arises, the vector of expected values lies exactly on the limit (sphere) of the tolerance ellipsoid. If the vector of expected values is situated outside of the tolerance region, the $E C_{p k}$ becomes negative. Thus the $E C_{p}$ and the $E C_{p k}$ must be interpreted similar to the univariate process capabilities $C_{p}$ and $C_{p k}$.

\subsection{Castagliola's Method}

Philippe Castagliola and Jose-Victor Garcia Castellanos proposed this method, in which the MPCI is based on the computation of the theoretical proportion of non-conforming proportion over convex polygons. Assuming the quality of a product depends on two characteristics $\left(X_{1}, X_{2}\right)$ and the corresponding tolerance limits are $\left[L_{1}, U_{1}\right]$ for $X_{1}$ and $\left[L_{2}, U_{2}\right]$ for $X_{2}$. These limits define a rectangular tolerance area called $A$. Then assuming $\boldsymbol{X}=\left(X_{1}, X_{2}\right)^{\mathrm{T}}$ is a bivariate normal random variable with expectance vector $\boldsymbol{\mu}=\left(\mu_{1}, \mu_{2}\right)^{T}$ and variance-covariance matrix $\Sigma=\mathrm{RVR}^{T}$ where

$$
\mathbf{R}=\left(\begin{array}{ll}
r_{1,1} & r_{2,1} \\
r_{1,2} & r_{2,2}
\end{array}\right) \text { and } \mathbf{V}=\left(\begin{array}{cc}
\sigma_{1}^{2} & 0 \\
0 & \sigma_{2}^{2}
\end{array}\right)
$$

The matrix $\mathrm{R}$ is a rotation matrix in which the unit eigenvectors $\mathbf{r}_{1}=\left(r_{1,1}, r_{1,2}\right)^{T}$ and $\mathbf{r}_{2}=\left(r_{2,1}, r_{2,2}\right)^{T}$ correspond to the main axis of the bivariate normal distribution. Then necessarily $r_{1,1}=r_{2,2}=\cos \theta$ and $r_{1,2}=-r_{2,1}=\sin \theta$, where $\theta$ is the rotation angle. The diagonal elements of matrix $\mathrm{V}$ are the variances on each main axis. In the sequel, it is assumed that $L_{1}<\mu_{1}<U_{1}$ and $L_{2}<\mu_{2}<U_{2}$. Let $D_{1}$ and $D_{2}$ be the straight lines passing through $\mu$ and having $r_{1}$ and $r_{2}$ as directions. These two lines split the $\left(X_{1}, X_{2}\right)$ plane into four regions $A_{1}, A_{2}, A_{3}$, and $A_{4}$. Because the bivariate normal distribution is symmetric respectively to the main axis, we necessarily have for $i=1,2,3,4$,

$$
P\left(X \in A_{i}\right)=1 / 4 \text {. }
$$

Let $Q_{1}, Q_{2}, Q_{3}, Q_{4}$ be the convex polygons defined as the intersection of regions $A_{1}, A_{2}, A_{3}, A_{4}$ and the rectangular tolerance area $A$ (i.e. $Q_{i}=A_{i} \cap A$ ), and let $P_{i}=A_{i}-Q_{i}$ be the complimentary region. Let $q_{i}=P\left(\mathrm{X} \in Q_{i}\right)$ and $p_{i}=P(\mathrm{X}$ $\left.\in P_{i}\right)$ be the probabilities that the random variable $\mathrm{X}=$ $\left(X_{1}, X_{2}\right)^{T}$ is respectively in $Q_{i}$ and $P_{i}$. Finally, let $q=P(\mathrm{X}$ $\in A$ ) be the probability that the random variable $\mathrm{X}$ is in the rectangular tolerance $A$ and $p=1-q$ be the total proportion of nonconforming products.

$$
p_{i}=1 / 4-q_{i}
$$

By definition, we clearly have $p=p_{1}+p_{2}+p_{3}+p_{4}$ and $q=$ $q_{1}+q_{2}+q_{3}+q_{4}$. From Eq. (18). we can deduce immediately
A new bivariate analogy for $C_{P K}$ called $B C_{P K}$ and defined as

$$
B C_{P K}=\frac{1}{3} \min \left\{-\Phi^{-1}\left(2 p_{1}\right),-\Phi^{-1}\left(2 p_{2}\right),-\Phi^{-1}\left(2 p_{3}\right)-\Phi^{-1}\left(2 p_{4}\right)\right\}
$$

We have used the following steps to estimate $B C_{P K}$ :

1. Estimating the expectance vector, $\mu$ and the variance-covariance matrix $\Sigma$ from the in-control sample,

$\hat{\Sigma}=\frac{1}{n-1} \sum_{k=1}^{n}\left(X_{k}-\hat{\mu}\right)\left(X_{k}-\hat{\mu}\right)^{T}$. and $\hat{\mu}=\frac{1}{n} \sum_{k=1}^{n} X_{k}$

2. Computing the eigenvalues (matrix $\hat{V}$ ) and eigenvectors (matrix $\hat{R}$ ) of $\hat{\Sigma}$,

3. Computing the vertices of the four convex polygons $Q_{1}, Q_{2}, Q_{3}$ and $Q_{4}$,

4. Using the method described at the last of this section computing the proportions $\hat{q}_{1}, \hat{q}_{2}, \hat{q}_{3}$ and $\hat{q}_{4}$. Derive $=\hat{p}_{i}=1 / 4-\hat{q}_{i}$ for $i=1, \ldots, 4$.

5. Computing the estimator for $B C_{P K}$

$B \hat{C}_{P K}=\frac{1}{3}\left\{-\Phi^{-1}\left(2 p_{1}\right),-\Phi^{-1}\left(2 p_{2}\right),-\Phi^{-1}\left(2 p_{3}\right),-\Phi^{-1}\left(2 p_{4}\right)\right\}$

\section{DATA COLLECTION}

Two quality characteristics (height and weight) of Can-cup S-9 were considered in this case and the data are collected from the Shinepukur Ceramic Industry.

Five hundred successive samples were taken. The specifications were defined as an interval for each variate: height $(4,6) \mathrm{cm}$. and weight $(190,242) \mathrm{gm}$., respectively; together they form a rectangular tolerance region. The center of the speciation was $\mu=(9,216)$ which was assumed to be the target of the process. The sample mean vector was $\overline{\mathbf{X}}=(5.55,219.73)$. Table 1 shows the statistical measures of the collected data.

The sample covariance matrix is

$$
\Sigma=\left[\begin{array}{cc}
0.45 & 0.46 \\
0.46 & 62.70
\end{array}\right]
$$

\section{RESULTS AND DISCUSSION}

\subsection{Test of statistical control}

As with all capability analyses, it is important that the process be in a statistical control when the capability is estimated. In the case of multivariate data, it is customary to use multivariate control chart like Hotelling's T-squared

\begin{tabular}{|c|c|c|}
\hline $\begin{array}{c}\text { Quality } \\
\text { Characteristics }\end{array}$ & Height & Weight \\
\hline$\sigma$ & 0.67 & 7.92 \\
\hline$\vec{\mu}_{\mathrm{o}}$ & 5 & 216 \\
\hline$\overline{\mathbf{X}}$ & 5.55 & 219.73 \\
\hline LSL $_{\mathrm{i}}$ & 4 & 190 \\
\hline $\mathrm{USL}_{\mathrm{i}}$ & 6 & 242 \\
\hline$\rho$ & & \\
\hline
\end{tabular}
control chart. Any T-squared value of the two variables for each sample beyond the upper control limit indicates a potentially out of control situation. Fig. 1 shows the process is in statistical control, where, upper control limit is 11.71 .

Table 1. Statistics of the collected data 


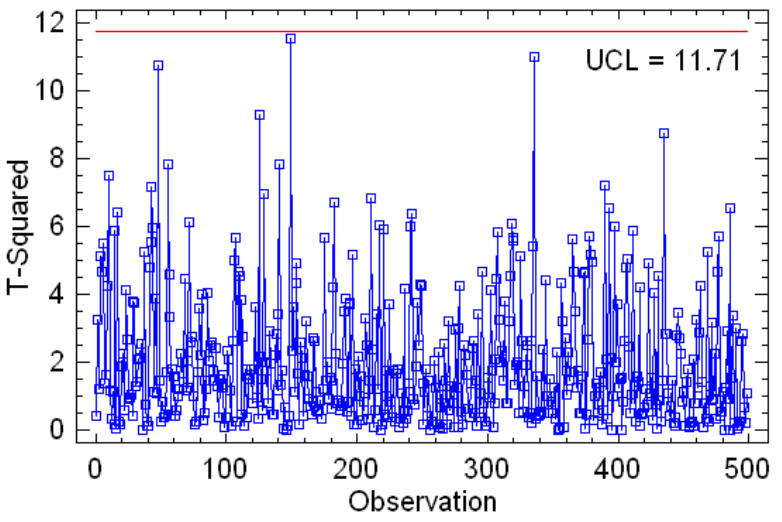

Fig. 1. Multivariate control chart

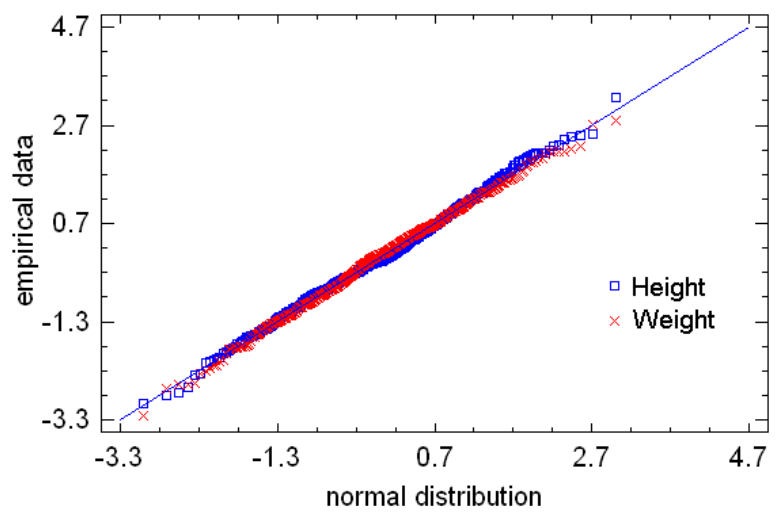

Fig. 2. Normal probability plot

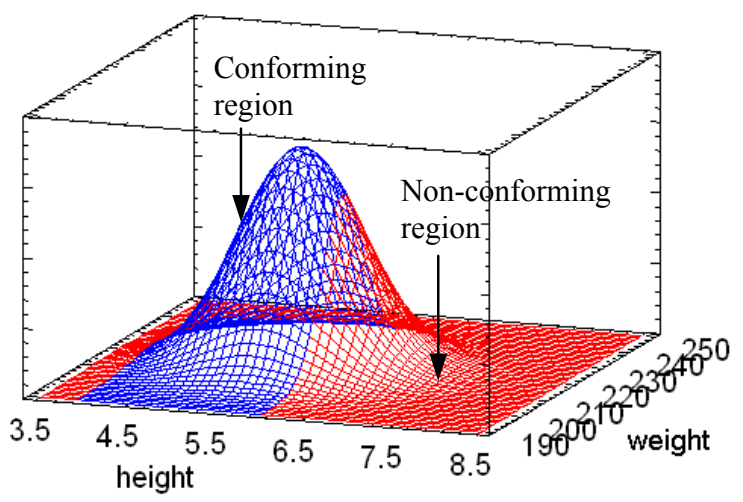

Fig. 3. Conforming and non-conforming regions in the bivariate normal distribution

\subsection{Test of normality}

This test is done to determine whether the variables can be adequately modeled by a normal distribution. Pvalues below 0.05 indicate significant departures from normality at the $5 \%$ significance level.

The following table provides P-values for each process.

Table 2. P-values for test of normality

\begin{tabular}{lcccc}
\hline & $\begin{array}{c}\text { Chi- } \\
\text { Squared }\end{array}$ & $\begin{array}{c}\text { Shapiro- } \\
\text { Wilk }\end{array}$ & Skewness & Kurtosis \\
\hline Height & 0.514938 & 0.538998 & 0.472998 & 0.49382 \\
Weight & 0.412758 & 0.516686 & 0.396079 & 0.662121
\end{tabular}

$\underline{\text { Table 3. Percentage out of specification observed }}$

\begin{tabular}{cc}
\hline Quality Variable & Beyond Specification \\
\hline Height & $24.6988 \%$ \\
Weight & $0.0 \%$ \\
Joint & $24.6988 \%$
\end{tabular}

From Table 2, we can see P-value for each test is above 0.05 . Since the values for both the variables are not less than 0.05 , there is not sufficient evidence to reject the hypothesis that the variables come from a normal distribution at the $5.0 \%$ significance level.

In the figure given above as all the data points falls approximately along the straight line, so the plot confirms that, the normal distribution is a reasonable model for this process.

\subsection{Capability plot}

Fig. 3. displays the fitted multivariate normal distribution for two variables. The area shaded in dark black corresponds to locations where all variables are within the specification limit. The area shaded in light black shows the region outside the specifications.

The observed percentage of quality variables out of specification both individually and jointly are provided in Table 3

\subsection{Comparison of multivariate capability indices}

Figure 4(a) illustrates the Taam's method's modified tolerance region centered on the target value. It can be noted that the Shahriari's method constructs the largest rectangle about the ellipse, while the Taam's method constructs the largest ellipse within the tolerance rectangle. The first component of Shahriari's method and the index of Taam's method are ratios related to the volumes of these respective modified regions.

Figure 4(b) illustrates the rectangular tolerance region for Chen's method. Target and process mean are labeled with their coordinates.

Figure 4(c) illustrates the tolerance region at the $99.73 \%$ process region (or ellipse) level, where the eigenvalues are 0.4464 and 62.7064; and the associated eigenvectors are $[-1,0.0074]$ and $[0.0074,1]$. The rectangle, drawn about the ellipse according to Shahriari's method, has labeled the modified process region.

Figure 4(d) illustrates the Braun's global approach to multivariate capability analysis. This is the only method which considers both the tolerance region and process region as an ellipse. The figure shows the process region is shifted from the tolerance region.

Figure 4(e) illustrates four convex polygons for Castagliola's method. The rotation matrix

$$
\left[\begin{array}{cc}
-1 & 0.0074 \\
0.0074 & 1
\end{array}\right]
$$

and vector of eigenvalues

$$
\left[\begin{array}{cc}
0.4464 & 0 \\
0 & 62.7064
\end{array}\right]
$$

calculated from the samples are used to draw two orthogonal lines passing through the process mean, which split the tolerance rectangle in four convex polygons. 


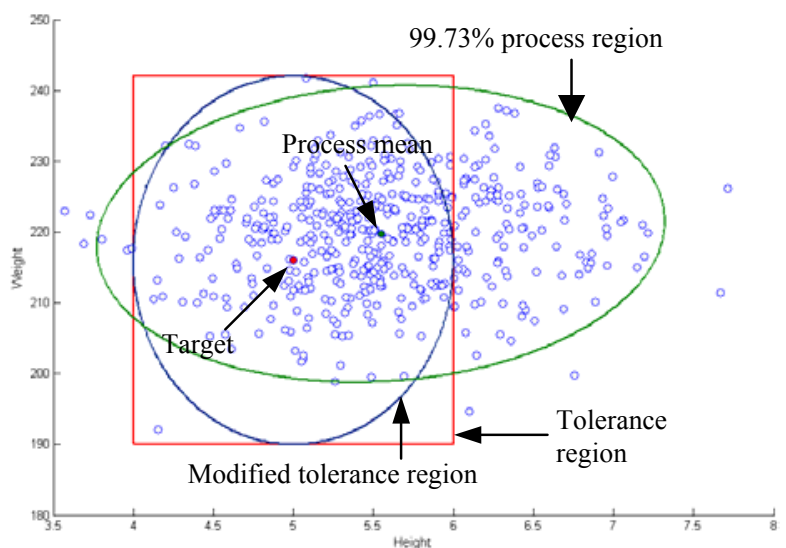

(a)

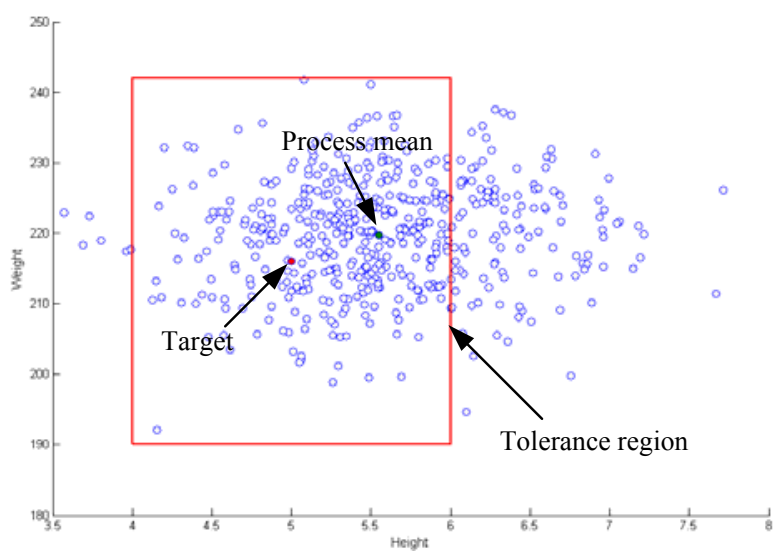

(b)

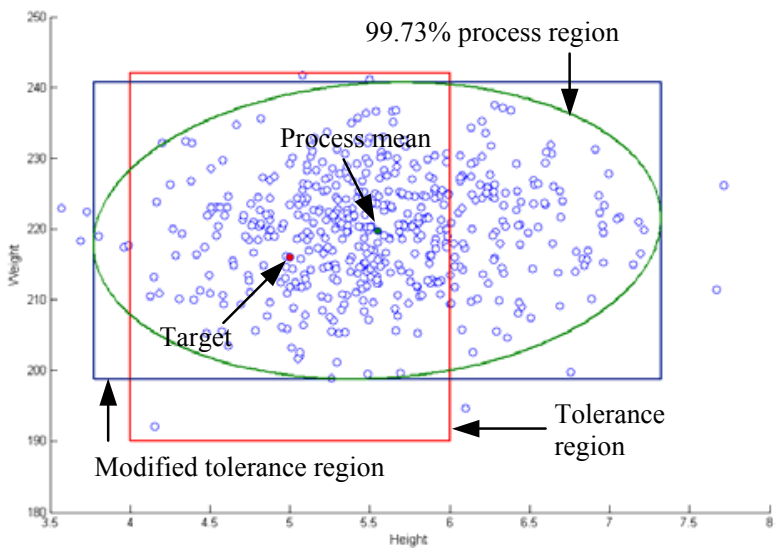

(c)

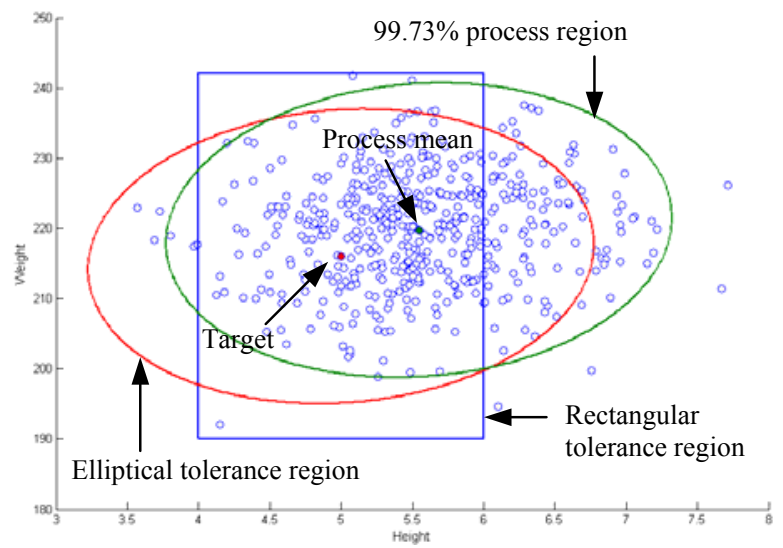

(d)

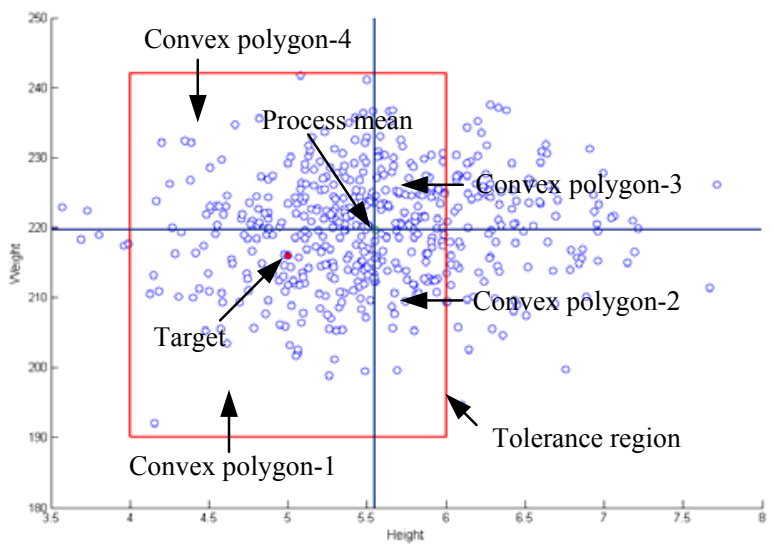

(e)

Figures: 4

(a) Geometry of tolerance and process region for Taam's method;

(b) Rectangular tolerance zone using Chen's method;

(c) Geometry of tolerance and modified process region for Shahriari's method;

(d) Elliptical tolerance and $99.73 \%$ process region by Braun's method; and

(e) Four convex polygons for Castagliola's method.
Table 4 shows how these indices differ with five different methods.

Table 4. MPCIs using five methods

\begin{tabular}{ccccc}
\hline Tam & Chen & Shahriari & Braun & $\begin{array}{c}\text { Castagl } \\
\text { iola }\end{array}$ \\
\hline$\underline{0.72}$ & 0.19 & {$[0.836,0.00,0]$} & $\mathrm{EC}_{\mathrm{p}}(1.04)$ & 0.22 \\
1.827 & $0.19 C_{\mathrm{pk}}(0.67)$ & 0.22 \\
$=0.39$ & & & &
\end{tabular}

In Taam's method as shown in Fig. 4(a) the modified tolerance ellipse is centered at the target as the largest ellipse about the tolerance rectangle. As it gives an index as a ratio of modified tolerance region to the $99.73 \%$ process region; it is expected that it must give a value less than 1. Because, from Fig. 4(a) we can see the process ellipse is bigger than the modified tolerance ellipse; not only that, one side of the process ellipse is out of the tolerance rectangle. The numerator $\hat{C}_{p}$ is smaller than 1 , 
which indicates that, the process variability is higher than allowed by the management. And the denominator is greater than 1, which implies the shift of the process mean from the target. As a consequence this method gives an index less than 1.

Another method detects this fact of shifting the process mean from the target is the second component of Shahriari's method. From Table 4 the second component is zero, implies that the process mean is off target.

Chen's method is based on probability of nonconformance. This method does not rely on the shift of the process mean from the specified target, as a result; the dispersion of the process mean from the target doesn't affect it. This index changes depending on the probability level and it takes in account only the tolerance rectangle. For this process, Fig. 4(b) also explains the matter that, the proportion of non-conforming products is high about the tolerance rectangle than defined by the management. This fact causes to give the index the value smaller than 1 .

As from Fig. 4(a), 4(c) and 4(d) it can be seen that, the left side of the 99.73 percent process ellipse exceeds the boundary of the original tolerance region. As Table 4 shows that the only method that clearly signals this situation is Shahriari's method, since the third component has value 0 . When this component is zero, it implies that the modified tolerance region extends beyond the tolerance region in at least one direction.

Further more; it is apparent that the second component of Shahriari's method and the index of Chen's method are sensitive to the region of acceptable product and the region of unacceptable product. In contrast, Taam's method modified tolerance regions only constrained to fit within these boundaries.

The index value of Shahriari's method, i.e. the first component, is close to 1 (Table 4), which suggests the variability of the process is not so bigger than allowed by the management. One cause of it is that, this index is the ratio of the area of the engineering tolerance region to the area of modified tolerance region. Fig. 4(c) shows that the area of the modified tolerance rectangle, i.e. the biggest rectangle about the process ellipse, is slightly bigger than the engineering tolerance rectangle, which causes the index to come closer to 1 , but not bigger than 1 .

Among all the five approaches, the only method which considers both the tolerance and modified tolerance region as an ellipse is Braun's method. Using elliptical tolerance region in the multivariate case, a precise control of processes becomes possible, considering the dependency structure of the quality characteristics. This means that products can be judged as a whole regarding the quality characteristics and not only as individual quality characteristics according to their specification, independently of all other quality characteristics.

Substantial elements of Braun's method are the elliptical capability indices $\mathrm{EC}_{\mathrm{p}}$ and $\mathrm{EC}_{\mathrm{pk}}$, which can be interpreted as the univariate capabilities $\mathrm{C}_{\mathrm{p}}$ and $\mathrm{C}_{\mathrm{pk}}$. Value of $\mathrm{EC}_{\mathrm{p}}$ is greater than 1, which implies the good capability of the process, but this index does not consider the shift of the process mean, as a result it can only justify that the process variability is less than allowed the management. On the other hand $\mathrm{EC}_{\mathrm{pk}}$ is lower than 1, which indicates the process mean is reasonably shifted off from the target, as also can be examined from Fig. 4(d).

As Chen's method, Castagliola also considers the proportion of non-conforming products but in a different way. First of all, it divides the tolerance rectangle in four convex polygons according to the eigenvalues and eigenvectors. Then it computes the theoretical proportion of non-conforming products over these convex polygons. Among the four convex polygons it takes only that value which one gives the minimum probability value for bivariate $B C_{P K}$, this is called as the exact way to compute the non-conforming proportion. This makes this index highly sensitive with the shift of process mean from the target.

From Fig. 4(e) it is easy to see how this method works, and its low index value gives a better estimation according to the management, and another reason to give such result accordingly is that, it takes into account $100 \%$ of the non-conforming zone, whereas others consider an elliptical region taking a smaller percentage of the tolerances.

In a summary, Castagliola's method gives the MPCI value which identifies the poor capability of this process as mentioned by the management. The management claimed the rejection rate as $30-40 \%$, whereas, this multivariate process capability study says the estimated rejection rate is $25.77 \%$. That is only $25.77 \%$ rejection will occur by this process.

The extra $6-10 \%$ rejection may be caused because of bad raw materials, or due to breakage while conveying within the industry or during packaging.

\section{CONCLUSION}

Five multivariate methods with different visions are applied in this paper for better understanding how to apply them in a ceramic industry and how much they are capable of assessing the capability of this ceramic cup manufacturing process. Hotelling's T-squared control chart is used to show the joint control for the two variables and status of statistical control. Bivariate normality is tested for validity of the four methods other than Chen's method. The multivariate process capability indices are computed and compared using selected methods to identify which one can best estimate the multivariate capability index for the considered process. All of the five methods show different estimation of the capability of the process. Among them, Castagliola's method shows it's consistency to give the MPCI value and rejection rate close to the management's claimed situation. The expected rejection rate is still somewhat less than the claim. The extra rejection is not caused by the process but by other causes related with man or materials involved with this manufacturing. Finally, this research work will provide an insight and guidance to the personnel involved in dealing with processes in similar manufacturing industries.

\section{ACKNOWLEDGEMENT}

Sincere gratitude is expressed to Shinepukur Ceramics Ltd., Beximco industrial park, Gazipur, for giving the privilege to perform multivariate process capability analysis in the Porcelain section of their ceramic industry.

\section{REFERENCES}

1. Taam, W., Subbiah, P. and W.Liddy, J., 1993, "A Note on Multivariate Capability Indices", Journal of Apllied Statistics, 20(3), pp. 339-351.

2. Chen, H., 1994, "A Multivariate Process Capability Index over a Rectangular Solid Tolerance Zone", Statistica Sinica, 4, pp. 749758. 
3. Shahriari, H., Hubele, N. F. and Lawrence, F. P., 1995, "A Multivariate Process Capability Vector", Proceedings of the $4^{\text {th }}$ Industrial Engineering Research Conference, pp. 303-308.

4. Braun, L., 2001, "New Methods in Multivariate Statistical Process Control (MSPC)", Diskussionsbeiträge des Fachgebietes Unternehmensforschung, pp. 1-12.

5. Castagliola, P. and Castellanos, J. G., 2005, "Capability Indices Dedicated to the Two Quality Characteristics Case", Quality Technology and Quantitative Management, 2(2), pp. 201-220.

6. Pal. S., 1999, "Performance Evaluation of a Bivariate Normal Process", Quality Engineering, 11(3), pp. 379-386.
7. Bothe, D. R., 1999, “Composite Capability Index for Multiple Product Characteristics", Quality Engineering, 12(2), pp. 253-258.

8. Wang, F. K. and Chen, J. C., 1998, "Capability Index using Principal Components Analysis", Quality Engineering, 11(1), pp. 21-27.

9. Wang, F. K. and Du, T. C. T., 2000, "Using Principal Component Analysis in Process Performance for Multivariate Data", Omega: The International Journal of Management Science, 28, pp. 185-194. 